\title{
Molecular cloning and expression analysis of a pearl oyster (Pinctada martensii) heat shock protein 90 (HSP90)
}

\author{
H.Y. Liang, Z.X. Wang, Q.N. Lei, R.L. Huang, Y.W. Deng, Q.H. Wang, Y. Jiao \\ and X.D. Du \\ Fisheries College, Guangdong Ocean University, Zhanjiang, China \\ Corresponding author: X.D. Du \\ E-mail: zjduxiaodong@163.com \\ Genet. Mol. Res. 14 (4): 18778-18791 (2015) \\ Received August 16, 2015 \\ Accepted October 4, 2015 \\ Published December 28, 2015 \\ DOI http://dx.doi.org/10.4238/2015.December.28.27
}

ABSTRACT. Heat shock protein 90 (HSP90) is an important molecular chaperone required for proper folding of cellular proteins, and thus, it plays an essential role in protecting cells from damage during stress. In this study, an HSP90 cDNA designated PmHSP90 was cloned from the mantle tissue of the pearl oyster Pinctada martensii using reverse transcription polymerase chain reaction (RT-PCR) coupled with the rapid amplification of cDNA ends (RACE) approach. PmHSP90 cDNA was 2584 bp in length, including an open reading frame of $2160 \mathrm{bp}$, which encodes a polypeptide of 719 amino acid residues, with predicted molecular mass and isoelectric point of $83.0 \mathrm{kDa}$ and 4.87 , respectively. Multiple-sequence alignment indicated that HSP9O is highly conserved among species, and PmHSP9O showed $89 \%$ sequence identity to Crassostrea gigas HSP90. Five conserved amino acid blocks defined as HSP90 protein family signatures were also observed in PmHSP90, indicating that PmHSP90 may be a cytosolic member of the HSP90 family. Expression levels of PmHSP90 were detected in various tissues of $P$. martensii and in hemocytes under three different stress conditions using quantitative real-time PCR (qPCR). The results demonstrate that PmHSP9O mRNA is constitutively expressed in 
all the tested tissues and may be involved in the immune response against thermal stress, lipopolysaccharide stimulation, and nucleus insertion operations. Studies on PmHSP90 are a valuable source to further explore the immune system in pearl oysters during the production of pearls, and may enhance our knowledge of molluscan innate immunity.

Key words: Heat shock protein 90 (HSP90); Pinctada martensii; Cloning; Expression; Stress

\section{INTRODUCTION}

Heat shock proteins (HSPs) are the most abundant, ubiquitously expressed, soluble, intracellular proteins and are phylogenetically conserved in all organisms (Srivastava, 2002). HSPs play crucial roles in response to potentially deleterious stress conditions, such as elevated temperatures, tissue trauma, heavy metal toxicity, radiation, and infection, or because of normal physiological alterations associated with cellular development and/or differentiation (Hartl, 1996; Kregel, 2002). Based on their molecular mass and function, HSPs can be divided into several families, such as HSP110, HSP90, HSP70, HSP60, HSP47, and low molecular mass HSPs (Lindquist and Craig, 1988). Of these families, HSP90 is a highly conserved molecular chaperone with a molecular mass of approximately $90 \mathrm{kDa}$, and is involved in protein folding, degradation, and signal transduction (Pearl and Prodromou, 2006). HSP90 represents 1 to $2 \%$ of the total cellular proteins in most tissues, making it one of the most abundant proteins, even in the absence of stress. The most well-known function of HSP90 includes the maintenance/folding of key proteins, such as steroid receptors and protein kinases, by forming specific complexes (Csermely et al., 1998). As an important member of the HSP family, HSP90 has recently received more attention in aquaculture research. In aquatic animals, HSP90 plays an important role in protecting organisms and can be induced by a range of stressors, such as reduced oxygen level (Li et al., 2009; Huang et al., 2014), heat or cold shock (Farcy et al., 2007; Jiang et al., 2009), hyperosmotic stress (Pan et al., 2000), food-deprivation (Cara et al., 2005), lipopolysaccharide (LPS) recognition (Tsutsui and Chung, 2012), heavy metals (Gao et al., 2007; Choi et al., 2008), and diseases (Wang et al., 2006; Chen et al., 2010). To date, many HSP90-encoding cDNAs have been described in bivalves, such as Crassostrea gigas (Choi et al., 2008), Chlamys farreri (Gao et al., 2007), Argopecten irradians (Gao et al., 2008), Crassostrea hongkongensis (Fu et al., 2011), Laternula elliptica (Kim et al., 2009), Mytilus galloprovincialis (Pantzartzi et al., 2009), and Ruditapes philippinarum (Liu et al., 2015). However, studies on pearl oyster (Pinctada martensii) HSP90 are rare, and the current knowledge of transcriptional expression levels of HSP9O under different stresses remains largely unknown.

The pearl oyster $P$. martensii is one of the most important species of molluscan mariculture, particularly in Southern China. P. martensii is primarily cultured for pearl production and the pearls produced by this species are known as "South China Sea Pearls" (Zhao et al., 2012). To obtain a cultured round pearl, a mantle graft cut from a donor oyster and a seed nucleus are implanted into the visceral mass of the host oyster. This process is known as nucleus insertion operation. However, the insertion of a nucleus into the visceral mass may increase the risk of infection. During the transplantation process, pearl oysters are prone to operational injury followed by bacterial infection (Ma et al., 2010). Moreover, diseases caused by various pathogens during breeding and cultivation practices occur frequently, leading to significant economic losses. In order to control disease and enhance the yields and quality of seawater pearls, it is necessary to further 
investigate the innate immune mechanisms in the pearl oyster.

In the current study, the full-length cDNA of heat shock protein 90 (designated as $\mathrm{PmH}$ SP90) was cloned from the mantle tissue of $P$. martensii using the rapid-amplification of cDNA ends (RACE) technique and then characterized by bioinformatics analysis. In addition, the expression profile of PmHSP9O in different tissues and under different stress conditions (i.e., thermal stress, LPS stimulation, and nucleus insertion operation) was investigated.

\section{MATERIAL AND METHODS}

\section{Animals and sample collection}

Pearl oysters approximately 2 years old and with a shell length ranging from 5 to $6 \mathrm{~cm}$ were directly collected from the sea in Chengwu, Zhanjiang, Guangdong Province, China. Nucleus insertion operations were performed at the Chengwu seawater pearl cultivation base. The immune stimulation experiments were performed in our laboratory. Animals were cultured at $25^{\circ}$ to $27^{\circ} \mathrm{C}$ in tanks with recirculating seawater for one week prior to the start of the experiments. Different tissues, including adductor muscles, mantle, hemocytes, hepatopancreas, gonads, and gills were collected from the pearl oysters and immediately stored in liquid nitrogen for further analyses. Among these tissues, the hemocytes were used for quantitative real-time PCR (qPCR) expression analyses. Hemolymph was collected from the adductor muscle sinus using a syringe and centrifuged at a rotor speed of $800 \mathrm{~g}$ for $10 \mathrm{~min}$ at $4^{\circ} \mathrm{C}$ to harvest hemocytes. Ten pearl oysters were randomly sampled at selected time points, detailed below.

\section{RNA extraction and cDNA synthesis}

Total RNA was extracted from tissues using TRIzol reagent (Invitrogen, USA) according to the manufacturer protocol. The extracted and purified total RNA was digested by RNase-free DNase (Promega, USA) to eliminate any DNA contamination. RNA quantity was measured using a NanoDrop 2000 spectrophotometer (Thermo, USA), while RNA integrity was assessed by electrophoresis on a $1 \%$ agarose gel containing ethidium bromide. cDNA was synthesized from $2 \mu \mathrm{g}$ total RNA using M-MLV reverse transcriptase (Promega) at $42^{\circ} \mathrm{C}$ for $60 \mathrm{~min}$ with oligo (dT) 18 primers following the manufacturer protocol.

\section{PCR and cloning of HSP90 cDNA}

Full-length HSP90 cDNA from $P$. martensii was obtained using the reverse transcription polymerase chain reaction (RT-PCR) and RACE technique. Primer pairs PmHSP90 01 and PmHSP90 02 (Table 1) were designed based on HSP9O unigenes, which were selected from the transcriptome library of the pearl sac following a nucleus insertion operation previously undertaken in our laboratory (Zhao et al., 2012). To obtain the intermediate fragment of PmHSP90, PCR was performed in an Eppendorf Mastercycler gradient (Eppendorf, German) thermal cycler, in a $25 \mu \mathrm{L}$ reaction volume containing $12.5 \mu \mathrm{L}$ Premix Taq, $1 \mu \mathrm{L}$ each primer, $9.5 \mu \mathrm{L}$ PCR-grade water, and 1 $\mu \mathrm{L}$ cDNA template. The PCR program was performed as follows: 5 min initial denaturation at $94^{\circ} \mathrm{C}$, followed by 32 cycles of denaturation at $94^{\circ} \mathrm{C}$ for $30 \mathrm{~s}$, annealing at $56^{\circ} \mathrm{C}$ for $40 \mathrm{~s}$, and elongation at $72^{\circ} \mathrm{C}$ for $1 \mathrm{~min}$, with a final extension step at $72^{\circ} \mathrm{C}$ for $10 \mathrm{~min}$ and cooling to $4^{\circ} \mathrm{C}$.

PCR products were separated on a $1 \%$ agarose gel and purified using a PCR purifica- 
tion kit (Promega). Purified PCR products were ligated into the pMD19-T vector (TaKaRa, Japan), and transformed into competent Escherichia coli cells DH5a. Bacteria were grown on ampicillincontaining Luria-Bertani (LB) plates and the recombinants were selected through blue-white color selection followed by screening with M13 forward and reverse primers (Table 1). Three positive clones were sequenced on an $\mathrm{ABI} 377$ automated sequencer ( $\mathrm{BBI}, \mathrm{USA}$ ), and the resulting sequences were verified and then subjected to cluster analysis.

\begin{tabular}{lll}
\multicolumn{2}{c}{ Table 1. Primer list used in this study. } & \\
\hline Primer name & Primer sequence (From 5'-3') & Application \\
\hline PmHSP90 01(forward) & CAAGCCAATTTGGACCCGTAA & Intermediate \\
PmHSP90 02(reverse) & AGTATTCATCGATGGGGTC & fragment PCR \\
PmHSP90 03(reverse) & CCACGCACAAAGTTCAAATACTCGGGAA & $5^{\prime}$ RACE \\
PmHSP90 04(reverse) & GGAATTCAAGCTGACCTTCCACTGAGA & $5^{\prime}$ RACE \\
PmHSP90 05(forward) & CGTGGTGTTGTAGACTCTGAGGACTTGC & 3' RACE \\
PmHSP90 06(forward) & TGGTATCCATGAAGATTCCACCAACAGG & $3^{\prime}$ RACE \\
UMP & CTAATACGACTCACTATAGGGC & RACE \\
NUP & AAGCAGTGGTATCAACGCAGAGT & RACE \\
PmHSP90 07(forward) & ATTTGAACTTTGTGCGTGG & qRT-PCR \\
PmHSP90 08(reverse) & AGCTTCTTCCTGTTGGTGG & qRT-PCR \\
Actin-F & CACTCGCCAAGATAATCAACG & qRT-PCR \\
Actin-R & CCATTCCTGTCAACTTCCCAT & qRT-PCR \\
M13-F & CGCCAGGGTTTTCCCAGTCACGAC & colony PCR \\
M13-R & GAGCGGATAACAATTTCACACAGG & colony PCR \\
\hline
\end{tabular}

The 5'-end of the PmHSP90 cDNA was obtained using a SMARTTM RACE cDNA Amplification kit (Clontech, USA). Two specific reverse primers, PmHSP90 03 and PmHSP90 04 (Table 1) were designed based on the above intermediate fragment sequence. PCR amplification was performed with cDNA template from mantle tissue RNA using the same reaction conditions as described above with a universal primer mix (UPM) (Table 1) and reverse primer PmHSP90 03, and then a nested PCR was carried out using a nested universal primer (NUP) and reverse primer PmHSP90 04. The 3'-end of PmHSP90 was amplified using the sense primer PmHSP90 05 and UMP (Table 1) and then a nested PCR was carried out using NUP and PmHSP90 06. All the amplified fragments were then sequenced.

\section{Bioinformatic analysis}

The identified PmHSP90 cDNA was analyzed for nucleotide sequence similarity with known sequences using the online NCBI BLAST tool (http://www.ncbi.nlm.nih.gov/BLAST/). The deduced amino acid sequence from PmHSP90 cDNA was analyzed using the Expert Protein Analysis System (http://www.expasy.org/). The open reading frame (ORF) of the cDNA was characterized using the online NCBI tool ORF Finder (http://www.ncbi.nlm.nih.gov/gorf/orfig.cgi). The molecular weight and theoretical isoelectric point $(\mathrm{pl})$ of the PmHSP90 cDNA encoded polypeptide were analyzed using the online tool ProtParam (http://web.expasy.org/protparam/). Multiple sequence alignment was performed with ClustalW1.83 (http://www.genome.jp/tools/clustalw/). Online tools SMART (http://smart.embl-heidelberg.de/) and PROSITE (http://prosite.expasy.org/) were used to predict domains and functional sites, respectively. A phylogenetic tree was constructed using the neighbor-joining method in MEGA 5.1 based on the nucleotide sequence homology between $\mathrm{Pm}$ HSP9O and other known HSP9O genes in the public databases. 


\section{Stress experiments using three different conditions}

For the thermal stress challenge, 60 pearl oysters were treated with a sublethal heat shock at $35^{\circ} \mathrm{C}$ for $1 \mathrm{~h}$ and were then transferred to $20^{\circ} \mathrm{C}$ for recovery. During recovery, a total of five time points, 3, 6, 12, 24, and $48 \mathrm{~h}$, were selected, with $0 \mathrm{~h}$ recorded as the control group.

For the lipopolysaccharide (LPS) challenge, 120 pearl oysters were randomly divided into two groups. The LPS challenge group was intramuscularly injected with $100 \mu \mathrm{L}(10 \mu \mathrm{g} / \mathrm{mL}) \mathrm{LPS}$ (Escherichia coli, 055:B5, Sigma) in phosphate buffered saline (PBS), while the control group was injected with $100 \mu \mathrm{L}$ PBS. Five post-injection time points, 3, 6, 12, 24, and $48 \mathrm{~h}$, were selected for expression analysis. Ten untreated pearl oysters were used as the control group $(0 \mathrm{~h})$.

The nucleus insertion operation was performed on 120 pearl oysters and a total of seven post operation time points, 1, 2, 3, 5, 10, 15, and 20 days were selected for expression analysis, with day 0 recorded as the control group.

\section{Quantitative analysis of PmHSP90 mRNA expression}

PmHSP90 mRNA expression was determined using qPCR with a gene-specific primer set (PmHSP90 07 and PmHSP90 08) and the $\beta$-actin gene as an internal reference (Table 1). The nucleotide sequences of the specific primers are provided in Table 1. Total RNA, from different tissues and hemocytes collected at different time points, was extracted using TRIzol (Invitrogen) and then treated with RNase-free DNase (Promega), as described above. A total of $2 \mu g$ RNA from each extracted sample was used as a template in RT-PCR to synthesize first-strand cDNA. A fluorescent real-time PCR assay was performed, using a Thermo Scientific DyNAmo Flash SYBR Green qPCR kit (Thermo) according to the manufacturer protocol, in an Applied Biosystems 7500 Fast Real-time system (ABI). qPCR was performed in 96-well plates and each sample was run in triplicate along with the reference gene. The amplification profile consisted of an initial denaturation step of $7 \mathrm{~min}$ at $95^{\circ} \mathrm{C}$ followed by 40 cycles each consisting of denaturation at $95^{\circ} \mathrm{C}$ for $15 \mathrm{~s}$, primer annealing at $57^{\circ} \mathrm{C}$ for $15 \mathrm{~s}$, and amplification at $72^{\circ} \mathrm{C}$ for $35 \mathrm{~s}$.

To assess the sensitivity and specificity of the assay, dissociation curve analysis of the amplification products was performed at the end of each run. After completion of all runs, data from the three replicates for each sample were analyzed using the 7500 System SDS software v2.0.1 (ABI). To maintain consistency, the baseline was automatically set by the software. Expression of $\mathrm{Pm}$ HSP90 was determined using the $2^{-\triangle \Delta C T}$ method (Livak and Schmittgen, 2001). Data obtained from qPCR analysis were presented as the means of $2^{-\Delta \Delta C T} \pm$ standard deviation (SD), followed by the Tukey's honestly significant difference (HSD) test to determine differences among the treatments. The differences were considered statistically significant at $P<0.05$. The data were analyzed using SPSS 19.0 software.

\section{RESULTS}

\section{cDNA cloning and sequencing of PmHSP90}

The intermediate PCR fragment amplified by primer sets 01 and 02 was $707 \mathrm{bp}$, and its nucleotide sequence was homogeneous to other known HSP90s. Based on the above sequence, two fragments of $P$. martensii HSP90 measuring 1066 bp and 1190 bp were amplified using 5'RACE and 3'-RACE, respectively. A 2584 bp nucleotide sequence, which was the full-length cDNA 
sequence of the PmHSP90 gene, was obtained through assembly of the three fragments amplified above. This sequence was deposited in GenBank under accession No. KF524260.1

\section{Characterization of PmHSP90}

PmHSP90 cDNA is 2584 bp long, including a 5'-untranslated region (5'-UTR) of $93 \mathrm{bp}$, a 3 '-UTR of $331 \mathrm{bp}$ with a canonical polyadenylation signal sequence AATAAA, and a poly(A) tail (Figure 1). The ORF of $2160 \mathrm{bp}$ encodes a polypeptide of 719 amino acid residues with a predicted molecular mass of $83.0 \mathrm{kDa}$ and a theoretical pl of 4.87. Five amino acid blocks defining the HSP90 protein family (NKEIFLRELISN[S/A/C]SDALDKIR, LGTIA[K/R]SGT, IGQFGVGFYSA[Y/F] LVA[E/D], IKLYVRRVFI, GVVDS[E/D]DLPLN[I/V]SRE), and the consensus sequence MEEVD at the C-terminus are highly conserved in the PmHSP90 sequence. SMART analysis revealed the location of a typical histidine kinase-like ATPase domain, which is ubiquitous in all HSP90 family members, from amino acid residues 30 to 184 (Figure 1).

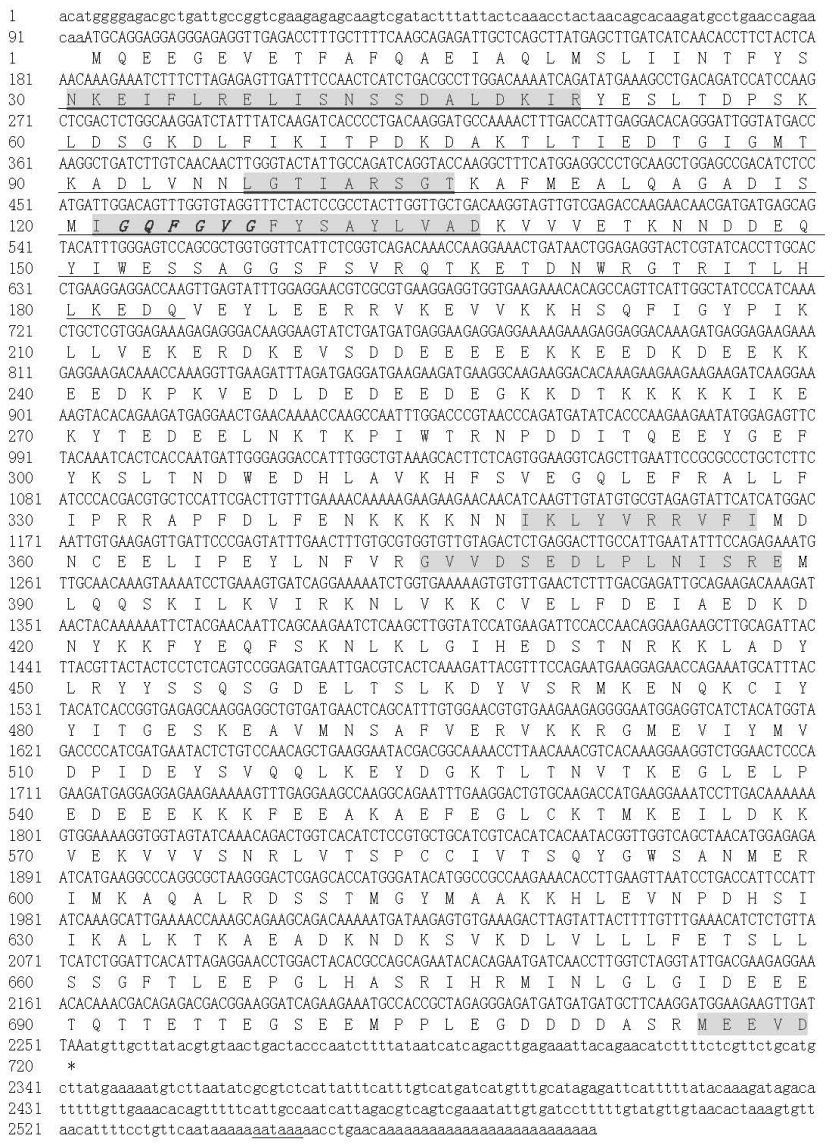

Figure 1. Nucleotide sequence and deduced amino acid sequence of the pearl oyster Pinctada martensii HSP90 CDNA. 5'UTR and 3'UTR are indicated with lowercase letters and open reading frame (ORF) region with capital letters. Five HSP90 family signatures and conserved "MEEVD" motif are shadowed. ATP binding domain and canonical polyadenylation signal sequence are underlined; conserved "GxxGxG" motif is bolded and italicized. 


\section{Homology analysis of PmHSP90}

The deduced amino acid sequence of PmHSP9O is highly similar to other HSP90s in invertebrates and vertebrates, especially in the regions of the HSP9O family signatures (Figure 2). It shares highest identity (89\%) with HSP90 from C. gigas, but it also shares $85 \%$ identity with HSP90 from A. irradians, 83\% with HSP90 from Cellana toreuma, and 81\% with HSP9O from $\mathrm{Ti}$ griopus japonicus. A phylogenetic tree, including PmHSP9O and other reported HSP9O genes, was constructed using MEGA 5.1 (Figure 3). Plant and animal HSP90s were separated into two distinct branches in the phylogenetic hierarchy. All the vertebrates were clustered together and formed two branches (HSP90 $\alpha$ and HSP90 $\beta$ isoform groups). All the vertebrates and arthropods were clustered together and formed a sister group to the branch of mollusks. There are two clear clades for bivalva and gastropoda sequences in the mollusk subgroup. PmHSP9O is closely related to the $H S P 90$ of bivalves. The relationships displayed in the phylogenetic tree were in good agreement with traditional taxonomy.

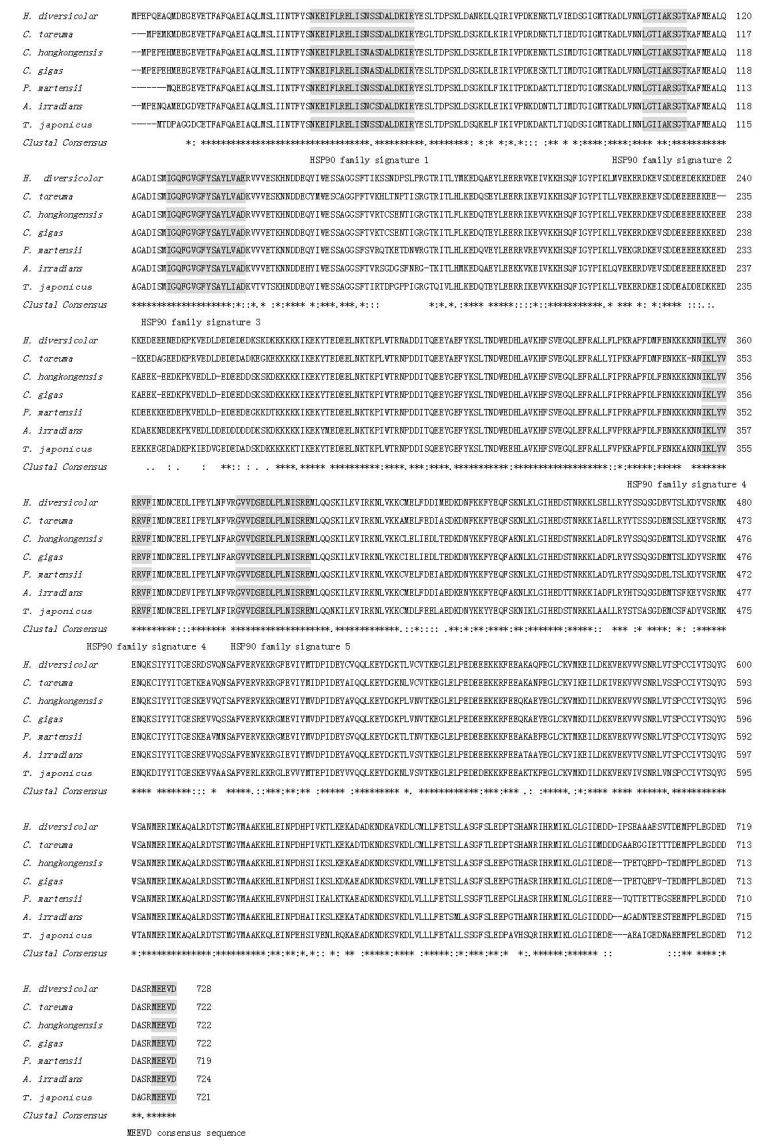

Figure 2. Multiple alignment of PmHSP90 with other species. HSP90 family signature sequences and consensus sequence "MEEVD" at the C-terminal are shaded with gray background. The symbols (asterisks, colons, and dots) in the ClustalW alignment format represent identical residue, conserved substitution, and semi-conserved substitution, respectively. 


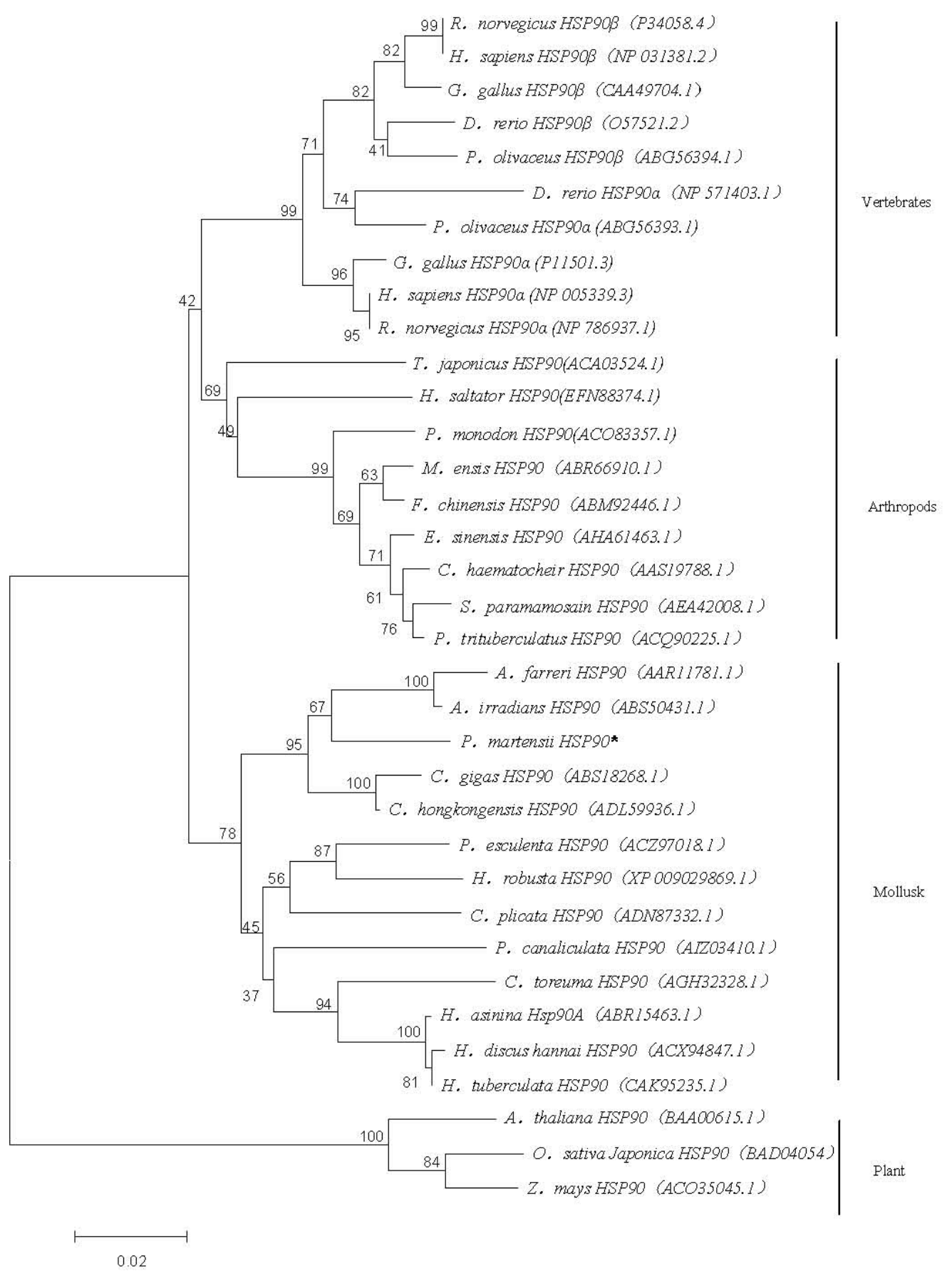

Figure 3. Phylogenetic analysis of PmHSP90. Phylogenetic tree of HSP90 was constructed with the neighbourjoinging method by the program MEGA 5.1. Numbers at each branch indicated the percentage bootstrap values. The GenBank accession numbers of all the HSP90 are indicated above. 


\section{Tissue distribution of PmHSP90 mRNA}

Expression of PmHSP90 mRNA was detected using qPCR in all the tested tissues, including adductor muscles, mantle, hemocytes, gonads, hepatopancreas, and gills (Figure 4). The highest PmHSP90 expression (10.15 fold of mantle) was observed in the hepatopancreas, followed by the hemocytes, mantle, and gonads. The lowest PmHSP90 expression was observed in the adductor muscles and gills.

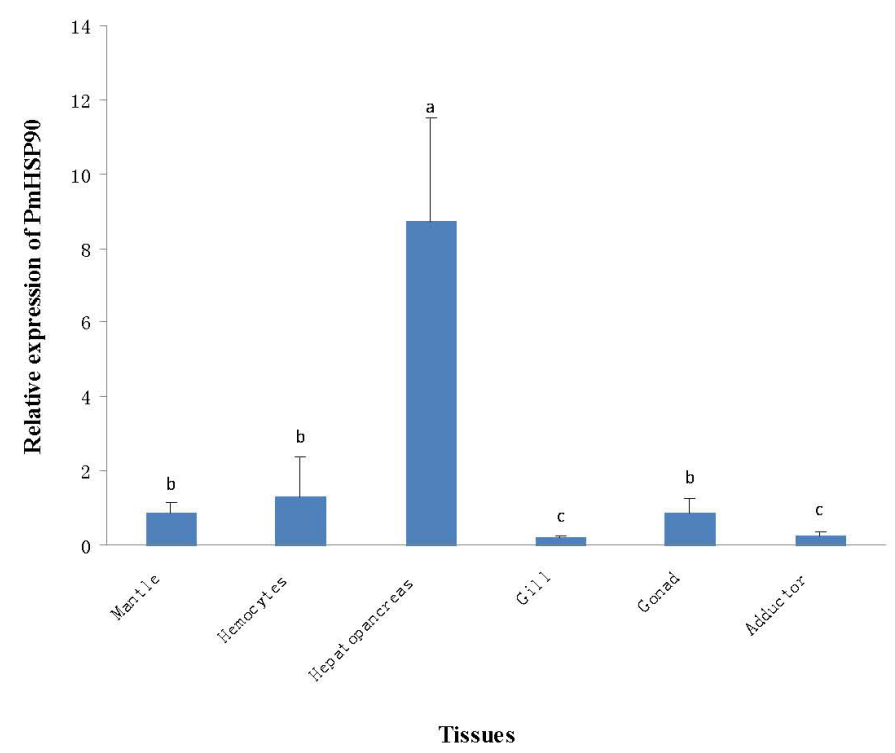

Figure 4. Tissue distribution of PmHSP90 mRNA. The relative mRNA expression of each tissue was compared to mantle expression to determine the tissue specific expression. Vertical bars represent the means \pm S.E.M. $(N=5)$. Significant difference was indicated by different letters $(P<0.05)$.

\section{Expression level of PmHSP90 transcripts in response to different stress conditions}

The temporal expression patterns of PmHSP9O in hemocytes under three different stress conditions are shown in Figure 5. Following exposure of $P$. martensii to heat shock at $35^{\circ} \mathrm{C}$ for $1 \mathrm{~h}, \mathrm{PmHSP90}$ expression levels were significantly up-regulated and maximum expression was reached at $3 \mathrm{~h}$ (28.89 times greater than the control group, $0 \mathrm{~h} ; \mathrm{P}<0.05)$. Expression levels then decreased gradually and attenuated to the original levels at $24 \mathrm{~h}$ post treatment (Figure 5A).

PmHSP90 expression was also examined in response to the LPS challenge. Post LPS injection, expression levels increased significantly and reached a peak at $3 \mathrm{~h}$, with 4.88 times greater expression compared to the control group ( $0 \mathrm{~h} ; P<0.05)$. Afterwards, the expression level decreased gradually and returned to the original level at the end of experiment (48 h; Figure 5B).

After the nucleus insertion operation, expression of PmHSP9O increased significantly and reached a peak at day 2 , with 7.47 times greater expression compared to the control group ( $0 \mathrm{~d} ; P$ $<0.05)$. Afterwards, the expression level of PmHSP90 tended to decrease gradually and returned to the original level at day 10 , with this level maintained until the end of the experiment (20 d; Figure $5 \mathrm{C})$. 
A)

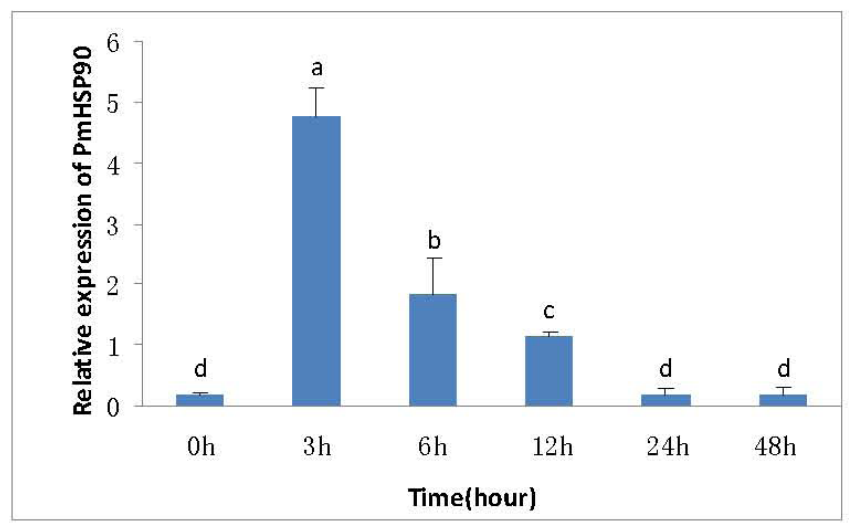

B)

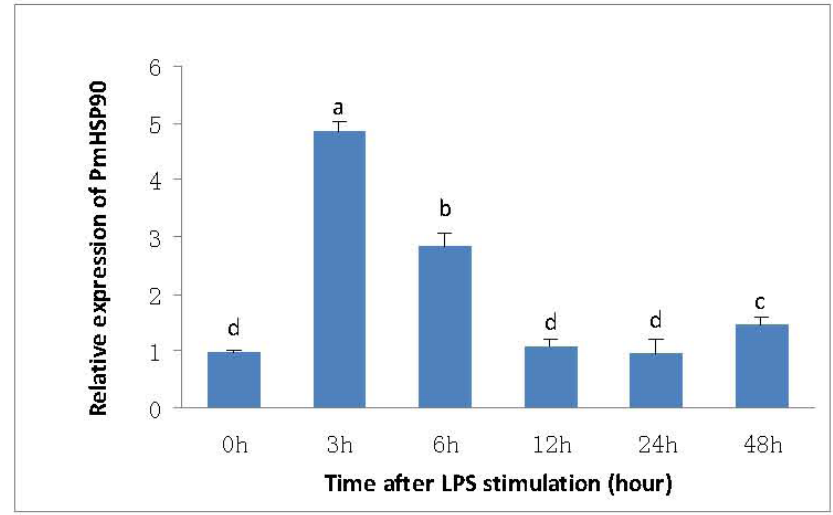

C)

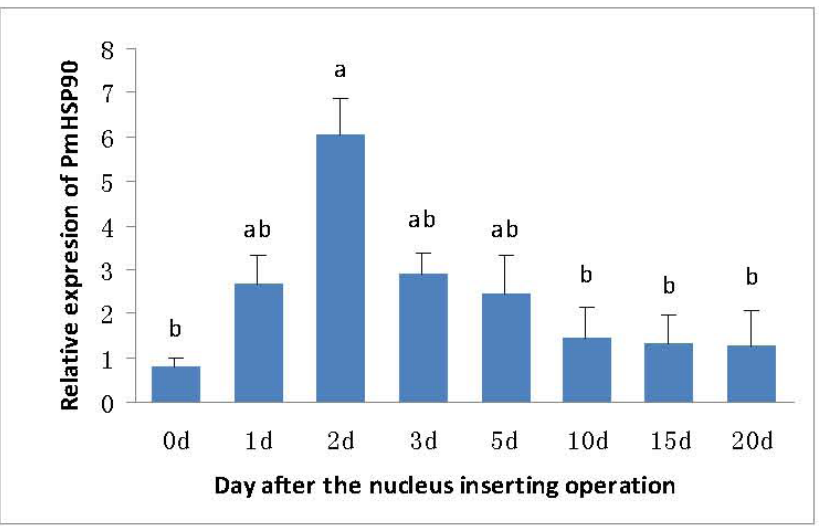

Figure 5. PmHSP90 expression profiles. (A) PmHSP90 expression in hemocytes after thermal stimulation. (B) PmHSP90 expression in hemocytes after LPS stimulation. (C) PmHSP90 expression in hemocytes after the nucleus inserting operation. The amount relative to the internal control is expressed as means \pm S.E.M. $(N=5)$. Significant difference was indicated by different letters $(P<0.05)$. 


\section{DISCUSSION}

P. martensii is one of the most important bivalve mollusks for seawater pearl production. Pearl formation is known to be induced by nucleus insertion into the visceral mass. However, the nucleus insertion operation may trigger a series of physiological responses, including an immune response, and induce injury and/or even death of the pearl oyster (Huang et al., 2015). Nucleus insertion injuries followed by bacterial infections have resulted in mass mortality of pearl oysters, resulting in heavy economic losses to the seawater pearl production industry (Miyazaki et al., 1999; Tomaru et al., 2001).

HSP90, a member of the heat shock protein family, is a ubiquitous molecular chaperone present in the cytoplasm of all the eukaryotic cells. In murine macrophages, HSP90 constitutes an essential component in several signal transduction pathways, including nuclear receptors for steroid hormones and various protein kinases (Zhu and Pisetsky, 2001). As a main component of immune responses, pearl oyster hemocytes are believed to be functionally analogous to vertebrate leukocytes and, therefore, play a crucial role in the recognition and removal of foreign materials (Kuchel et al., 2010). There is copious evidence that the HSP90 found in hemocytes is associated with the stress response (Gao et al., 2007; Gao et al., 2008; Araya et al., 2010; Fu et al., 2011). Therefore, cloning of the full length HSP90 cDNA, followed by analysis of its expression profiles in hemocytes after immune stimulation and the nucleus insertion operation, can contribute to the development of novel strategies for mass control of aquaculture mortality.

In the present study, a new full-length HSP90 cDNA was isolated and sequenced from P. martensii. Conserved sequences and characteristic motifs, such as HSP90 family signatures, ATP, and the geldanamycin binding domain, were found in the deduced PmHSP90 amino acid sequences, consistent with previous studies (Chio et al., 2008). It has been reported that this peptide is recognized by the tetratricopeptide repeat (TPR) domains of HOP (HSP70 and HSP90 organizing protein), which is an adapter protein that mediates the association of HSP70 and HSP90 into a multi-chaperone complex (Scheufler et al., 2000). Based on the presence of an MEEVD sequence at its C-terminus, PmHSP90 was concluded to be a member of the cytosolic HSP90 family.

Homology analysis revealed that the deduced amino acid sequence of PmHSP90 shares a high similarity with known HSP90s. The sequence alignment and phylogenetic analysis further suggest that PmHSP9O is a member of the HSP90 family of chaperones. In the phylogenetic tree, PmHSP90 is located on the branch of mollusks and is closely related to the bivalves. In vertebrates, two different isoforms of HSP90, HSP90 $\alpha$ and HSP90 $\beta$, exist. Like all vertebrate HSP90 $\beta$-isoforms, PmHSP90 lacks the glutamine-rich sequence (QTQDQ) at the N-terminus, which is a site of phosphorylation by a dsDNA-dependent kinase (Theodoraki and Mintzas, 2006) and is a characteristic of all $\alpha$-isoforms. This suggests that $P m H S P 90$ is more closely related to the vertebrate HSP90 $\beta$-isoforms (Gao et al., 2008).

The expression patterns of PmHSP90 were investigated in order to better understand its physiological functions. Under normal conditions, PmHSP9O was constitutively expressed in all the examined tissues, though transcript levels varied in different tissues. This indicates that HSP90 plays a key role as a ubiquitously expressed factor in $P$. martensii, and is involved in a wide array of cellular processes. Following sublethal heat shock treatment, the relative expression of PmHSP9O was significantly up-regulated, reaching a peak at $3 \mathrm{~h}$. This heat-inducible property confirms that PmHSP90 is an inducible HSP family member. With a recovery time of $48 \mathrm{~h}$, the expression level of the PmHSP90 gene gradually attenuated to the original level. The rapid and reversible induction of the PmHSP90 gene in response to the heat shock demonstrated its innate advantages in tracing thermal stressors. The results are almost in agreement with previous findings in disk abalone (Far- 
cy et al., 2007; Wang et al., 2011) and shrimp (Jiang et al., 2009; Li et al., 2009). These data suggest that $H S P 90$ may be directly involved in the heat shock resistance mechanisms in different organisms.

Besides the heat shock response, LPS challenge also triggered significant induction of PmHSP90. Within $48 \mathrm{~h}$ of the LPS injection, the relative expression of PmHSP90 significantly increased and reached maximum levels at $3 \mathrm{~h}$. This indicates that the underlying induction mechanism may perhaps resemble those in other animal species. In mammals, it has been demonstrated that HSP90 may directly bind to LPS as a receptor in the recognition of innate immunity and the signaling pathway of the inflammatory response (Triantafilou et al., 2001; Triantafilou and Triantafilou, 2004). In marine animals, in response to LPS challenge, the transcription of the disk abalone HSP90 gene significantly increased within $2 \mathrm{~h}$ and approached maximum induction at $4 \mathrm{~h}$, recovering to the original level in $24 \mathrm{~h}$ (Wang et al., 2011). LPS challenge also caused a maximum 55 fold increase in HSP90 expression in blue crabs (Tsutsui and Chung, 2012). Moreover, being an endotoxin, LPS may damage the cellular proteins, thereby up-regulating HSP9O expression. Nevertheless, the results of the current study suggest that PmHSP9O is sensitive to the invasion of LPS in the pearl oyster. HSP90 is a general molecular chaperone involved in the correct folding and processing of a variety of cellular regulators (Hartl, 1996). The provoked increase in HSP90 expression was perhaps a protection approach of the organisms from further damage.

Furthermore, the results of the nucleus insertion operation indicate that PmHSP9O plays a vital role in wound healing and immune response to the foreign body (the inserted nucleus). Similarly, expression of the NFAT gene was observed to increase after nucleus insertion in $P$. martensii (Huang et al., 2015) and expression of the a2M gene was also found to be up-regulated after nucleus insertion in the pearl oyster Hyriopsis cumingii Lea (Li et al., 2010). These results are valuable for further exploring the immune response of the host oyster after nucleus implantation.

In conclusion, PmHSP90 was cloned from the pearl oyster P. martensii. Comparison and phylogenetic analysis revealed that $P m H S P 90$ shows a relatively high similarity with other invertebrate and vertebrate HSP90s. This study indicates that PmHSP90 mRNA is constitutively expressed in various tissues. Furthermore, gene expression analysis revealed that the HSP9O is involved in the immune response to thermal stress, LPS stimulation, and injury during nucleus insertion. Future studies on PmHSP90 expression will help in further exploring the immune response in pearl oysters during the production of pearl and may enhance our knowledge and understanding of the molluskan innate immunity.

\section{Conflicts of interest}

The authors declare no conflict of interest.

\section{ACKNOWLEDGMENTS}

Research supported by the National Natural Science Foundation of China (\#31472306), the Science and Technology Program of Guangdong, China (\#2012A031100010), and the Science and Technology Program of Zhanjiang, China (\#2013A03022).

\section{REFERENCES}

Araya MT, Markham F, Mateo DR, McKenna P, et al. (2010). Identification and expression of immune-related genes in hemocytes of soft-shell clams, Mya arenaria, challenged with Vibrio splendidus. Fish Shellfish Immunol. 29: 557-564. 
Cara JB, Aluru N, Moyano FJ and Vijayan MM (2005). Food-deprivation induces HSP70 and HSP90 protein expression in larval gilthead sea bream and rainbow trout. Comp. Biochem. Physiol. B-Biochem. Mol. Biol. 142: 426-431.

Chen YM, Kuo CE, Wang TY, Shie PS, et al. (2010). Cloning of an orange-spotted grouper Epinephelus coioides heat shock protein 90AB (HSP90AB) and characterization of its expression in response to nodavirus. Fish Shellfish Immunol. 28: 895-904.

Choi YK, Jo PG and Choi CY (2008). Cadmium affects the expression of heat shock protein 90 and metallothionein mRNA in the Pacific oyster, Crassostrea gigas. Comp. Biochem. Physiol. C-Toxicol. Pharmacol. 147: 286-292.

Csermely P, Schnaider T, Soti C, Prohaszka Z, et al. (1998). The 90-kDa molecular chaperone family: structure, function, and clinical applications. A comprehensive review. Pharmacol. Ther. 79: 129-168.

Farcy E, Serpentini A, Fievet B and Lebel JM (2007). Identification of cDNAs encoding HSP70 and HSP90 in the abalone Haliotis tuberculata: transcriptional induction in response to thermal stress in hemocyte primary culture. Comp. Biochem. Physiol. B-Biochem. Mol. Biol. 146: 540-550.

Fu D, Chen J, Zhang Y and Yu Z (2011). Cloning and expression of a heat shock protein (HSP) 90 gene in the haemocytes of Crassostrea hongkongensis under osmotic stress and bacterial challenge. Fish Shellfish Immunol. 31: 118-125.

Gao Q, Song L, Ni D, Wu L, et al. (2007). cDNA cloning and mRNA expression of heat shock protein 90 gene in the haemocytes of Zhikong scallop Chlamys farreri. Comp. Biochem. Physiol. B-Biochem. Mol. Biol. 147: 704-715.

Gao Q, Zhao J, Song L, Qiu L, et al. (2008). Molecular cloning, characterization and expression of heat shock protein 90 gene in the haemocytes of bay scallop Argopecten irradians. Fish Shellfish Immunol. 24: 379-385.

Hartl FU (1996). Molecular chaperones in cellular protein folding. Nature 381: 571-579.

Huang XD, Wei GJ, Zhang $\mathrm{H}$ and He MX (2015). Nuclear factor of activated T cells (NFAT) in pearl oyster Pinctada fucata: molecular cloning and functional characterization. Fish Shellfish Immunol. 42: 108-113.

Huang YT, Cai XH, Zou ZH, Wang SH, et al. (2014). Molecular cloning, characterization and expression analysis of three heat shock responsive genes from Haliotis diversicolor. Fish Shellfish Immunol. 36: 590-599.

Jiang S, Qiu L, Zhou F, Huang J, et al. (2009). Molecular cloning and expression analysis of a heat shock protein (HSP90) gene from black tiger shrimp (Penaeus monodon). Mol. Biol. Rep. 36: 127-134.

Kim M, Ahn IY, Kim H, Cheon J, et al. (2009). Molecular characterization and induction of heat shock protein 90 in the Antarctic bivalve Laternula elliptica. Cell Stress Chap. 14: 363-370.

Kregel KC (2002). Heat shock proteins: modifying factors in physiological stress responses and acquired thermotolerance. J. Appl. Physiol. 92: 2177-2186.

Kuchel RP, Raftos DA, Birch D and Vella N (2010). Haemocyte morphology and function in the Akoya pearl oyster, Pinctada imbricate. J. Invertebr. Pathol. 105: 36-48.

Li F, Luan W, Zhang C, Zhang J, et al. (2009). Cloning of cytoplasmic heat shock protein 90 (FcHSP90) from Fenneropenaeus chinensis and its expression response to heat shock and hypoxia. Cell Stress Chap. 14: 161-172.

Li W, Shi Z and He X (2010). Study on immune regulation in Hyriopsis cumingii Lea: effect of pearl-nucleus insertion in the visceral mass on immune factors present in the hemolymph. Fish Shellfish Immunol. 28: 789-794.

Lindquist S and Craig EA (1988). The heat-shock proteins. Annu. Rev. Genet. 22: 631-677.

Liu T, Pan LQ, Cai YF and Miao JJ (2015). Molecular cloning and sequence analysis of heat shock proteins 70 (HSP70) and 90 (HSP90) and their expression analysis when exposed to benzo(a)pyrene in the clam Ruditapes philippinarum. Gene 555: 108-118.

Livak KJ and Schmittgen TD (2001). Analysis of relative gene expression data using real-time quantitative PCR and the 2(-Delta Delta C(T)) method. Methods 25: 402-408.

Ma J, Zhang D, Jiang J, Cui S, et al. (2010). Molecular characterization and expression analysis of cathepsin L1 cysteine protease from pearl oyster Pinctada fucata. Fish Shellfish Immunol. 29: 501-507.

Miyazaki T, Goto K, Kobayashi T, Kageyama T, et al. (1999). Mass mortalities associated with a virus disease in Japanese pearl oysters Pinctada fucata martensii. Dis. Aquat. Org. 37: 1-12.

Pan F, Zarate JM, Tremblay GC and Bradley TM (2000). Cloning and characterization of salmon hsp90 cDNA: upregulation by thermal and hyperosmotic stress. J. Exp. Zool. 287: 199-212.

Pantzartzi CN, Kourtidis A, Drosopoulou E, Yiangou M, et al. (2009). Isolation and characterization of two cytoplasmic hsp90s from Mytilus galloprovincialis (Mollusca: Bivalvia) that contain a complex promoter with a p53 binding site. Gene 431: 47-54.

Pearl LH and Prodromou C (2006). Structure and mechanism of the Hsp90 molecular chaperone machinery. Annu. Rev. Biochem. 75: 271-294.

Scheufler C, Brinker A, Bourenkov G, Pegoraro S, et al. (2000). Structure of TPR domain-peptide complexes: critical elements in the assembly of the Hsp70-Hsp90 multichaperone machine. Cell 101: 199-210.

Srivastava P (2002). Roles of heat-shock proteins in innate and adaptive immunity. Nat. Rev. Immunol. 2: $185-194$.

Theodoraki MA and Mintzas AC (2006). cDNA cloning, heat shock regulation and developmental expression of the hsp83 gene in the Mediterranean fruit fly Ceratitis capitata. Insect Mol. Biol. 15: 839-852. 
Tomaru Y, Kawabata Z and Nakano S (2001). Mass mortality of the Japanese pearl oyster Pinctada fucata martensii in relation to water temperature, chlorophyll a and phytoplankton composition. Dis. Aquat. Org. 44: 61-68.

Triantafilou K, Triantafilou M and Dedrick RL (2001). A CD14-independent LPS receptor cluster. Nat. Immunol. 2: 338-345.

Triantafilou M and Triantafilou K (2004). Heat-shock protein 70 and heat-shock protein 90 associate with Toll-like receptor 4 in response to bacterial lipopolysaccharide. Biochem. Soc. Trans. 32: 636-639.

Tsutsui $\mathrm{N}$ and Chung JS (2012). A novel putative lipoprotein receptor (CasLpR) in the hemocytes of the blue crab, Callinectes sapidus: cloning and up-regulated expression after the injection of LPS and LTA. Fish Shellfish Immunol. 32: 469-475.

Wang B, Li F, Dong B, Zhang X, et al. (2006). Discovery of the genes in response to white spot syndrome virus (WSSV) infection in Fenneropenaeus chinensis through cDNA microarray. Mar. Biotechnol. 8: 491-500.

Wang N, Whang I, Lee JS and Lee J (2011). Molecular characterization and expression analysis of a heat shock protein 90 gene from disk abalone (Haliotis discus). Mol. Biol. Rep. 38: 3055-3060.

Zhao XX, Wang QH, Jiao Y, Huang RL, et al. (2012). Identification of genes potentially related to biomineralization and immunity by transcriptome analysis of pearl sac in pearl oyster Pinctada martensii. Mar. Biotechnol. 14: 730-739.

Zhu FG and Pisetsky DS (2001). Role of the heat shock protein 90 in immune response stimulation by bacterial DNA and synthetic oligonucleotides. Infect. Immun. 69: 5546-5552. 